\title{
Dynamic expression and roles of sequestome-1/p62 in LPS-induced acute kidney injury in mice
}

\author{
TING LI $^{1,2^{*}}$, JIE ZHAO $^{3 *}$, SHUYING MIAO $^{1}$, YUNFEI XU $^{1}$, XIANZHONG XIAO $^{1}$ and YING LIU ${ }^{1}$ \\ ${ }^{1}$ Department of Pathophysiology, Xiangya School of Medicine, Central South University, Changsha, Hunan 410078; \\ ${ }^{2}$ Department of Physiology, Changzhi Medical College, Changzhi, Shanxi 046000; ${ }^{3}$ Department of Neurosurgery, \\ Xiangya Hospital, Central South University, Changsha, Hunan 410078, P.R. China
}

Received April 5, 2017; Accepted September 6, 2017

DOI: $10.3892 / \mathrm{mmr} .2018 .8809$

\begin{abstract}
Acute kidney injury (AKI) is one of the most common complications of sepsis. The roles of autophagy in AKI have been demonstrated in previous studies. Sequestosome-1 (p62) has been demonstrated to serve essential roles in autophagy. The dysregulation of autophagy causes p62 accumulation, which is associated with increased inflammation and tumorigenesis. However, the expression patterns and role of p62 in septic AKI remain unknown. The present study detected the renal autophagy level, and the expression and localization of p62, in a lipopolysaccharide (LPS)-induced AKI mouse model. The results demonstrated that autophagy was induced in the kidneys of LPS-treated mice. The mRNA and protein levels of p62 were decreased in whole renal tissue samples and increased in mice treated with LPS. Immunohistochemistry indicated that $\mathrm{p} 62$ protein was predominantly expressed in the cytoplasm of proximal tubules under normal conditions and was significantly decreased following LPS injection into the cortex. In addition, p62 protein was gradually redistributed to the outer and inner medullas following treatment with LPS. In vitro experiments demonstrated that overexpression of p62 significantly decreased the viability and increased the lactate dehydrogenase (LDH) release and apoptosis rate, of renal tubular epithelial cells. By contrast, interference with p62 expression using small interfering RNA increased the cell viability and decreased the LDH release and apoptosis rate. The results of the present study demonstrated that p62
\end{abstract}

Correspondence to: Dr Xianzhong Xiao or Dr Ying Liu, Department of Pathophysiology, Xiangya School of Medicine, Central South University, 110 Xiangya Road, Changsha, Hunan 410078, P.R. China

E-mail: xiaoxianzhong@csu.edu.cn

E-mail: liu1977ying@126.com

${ }^{*}$ Contributed equally

Key words: sequestome-1, acute kidney injury, sepsis, endotoxemic mice, lipopolysaccharide may aggravate LPS-induced acute kidney injury in mice by promoting apoptosis in renal tubular epithelial cells.

\section{Introduction}

Acute kidney injury (AKI) is one of the most common and serious complications of sepsis (1). The morbidity of AKI secondary to sepsis in the intensive care unit may reach $70 \%$ (2). The mortality rate for septic patients with AKI is increased compared with patients with sepsis alone (3). However, there are no effective therapeutic strategies to treat this disease. Therefore, a better understanding of the molecular basis of the disease may contribute to more targeted therapies of septic AKI.

Autophagy is a protective mechanism of the body against injury, which may maintain the homeostasis of the body (4). Recent studies have demonstrated that autophagy is activated during AKI $(5,6)$. Leventhal et al (7), reported that autophagy may be induced by lipopolysaccharide (LPS) in renal tubular epithelial cells, and that the induction of autophagy has a protective effect in AKI. As a selective autophagy signaling adaptor, sequestosome-1 (p62) is one of the indicators of the level of autophagy $(8,9)$. The ablation of important autophagy proteins has been observed to increase p62 levels and to damage renal function. (6) p62 possesses a number of important domains that facilitate interactions with numerous signaling activators $(10,11)$. It has been demonstrated that p62-mediated selective autophagy is regulated by its PB1 domain, light chain (LC) 3-interacting region and ubiquitin-associated domain (12). The N-terminal PB1 domain enables p62 to self-associate and polymerize in the cytoplasm to form aggregates and cytoplasmic inclusion bodies, and these self-aggregates are degraded by autophagy (13). The C-terminal ubiquitin-binding domain enables p62 to recognize and bind to polyubiquitinated proteins (11). The LC3-interacting region (LIR) facilitates p62 binding to LC3-II (a marker of autophagy) and p62-bound polyubiquitinated cargo to the autophagosomes for degradation (14). The selective turnover of p62 by autophagy serves an important role in the formation of cytoplasmic proteinaceous aggregates, identified as a common hallmark of autophagy-deficient post-mitotic cells $(8,15)$. Since p62 is implicated in a number of signaling pathways, its expression level is important. Recently, p62 has 
been demonstrated to serve essential roles in various diseases, including liver and breast cancer $(16,17)$, Paget's disease of bone $(18)$, obesity and insulin resistance $(19,20)$. However, little is known about the expression pattern and roles of p62 in septic AKI.

In the present study, a mouse model of endotoxemia was developed via intraperitoneal injection of LPS, the most common toxemia model. Kidney tissue was used to detect the expression and location of p62. In addition, at the cellular level, the present study overexpressed and knocked down p62 in renal tubular epithelial cells by transfection of p62 overexpression plasmids and siRNA, respectively, to analyze the possible roles.

\section{Materials and methods}

Animals and treatment. Male C57BL/6 mice at 8 weeks of age, 20 25 g, were purchased from the Experimental Animal Center of Central South University (Changsha, China), and housed in a temperature $\left(25^{\circ} \mathrm{C}\right)$ and humidity $(40 \sim 60 \%)$ controlled facility on a 12 -h light/dark cycle with free access to food and water. A total of 30 mice were divided into two groups: The LPS group $(\mathrm{n}=20)$, injected with LPS $(10 \mathrm{mg} / \mathrm{kg}$; intraperitoneal); and the control group $(n=10)$, injected with saline. Subsequently, the 72-h survival rate was observed. An additional 40 mice were divided into five groups $(n=8)$ and injected with LPS (10 mg/kg; intraperitoneal) for 0 (control), 4, 8, 12 and $24 \mathrm{~h}$ individually. The mice were anesthetized and bled by heart puncture. Subsequently, the mice were sacrificed, upper and lower parts of the left kidneys were collected in liquid nitrogen and then stored at $-80^{\circ} \mathrm{C}$ for reverse transcription-quantitative polymerase chain reaction (RT-qPCR) analysis and western blotting. Middle parts of the left kidneys were collected and fixed in $4 \%$ paraformaldehyde at $4^{\circ} \mathrm{C}$ overnight for paraffin embedded and $3 \mu \mathrm{m}$ sections were cut and stained with immunohistochemistry. The cortex and medulla of the right kidneys were separated and collected in liquid nitrogen and then stored at $-80^{\circ} \mathrm{C}$ until for western blotting analysis. All animal experimental protocols were approved by the institutional ethics committee for animal experiments of Central South University.

Assessment of renal function. Renal functional parameters including blood urine nitrogen (BUN) and creatinine were detected using a Synchron CX7 autoanalyzer (Beckman Coulter, Inc., Brea, CA, USA).

Reverse-transcription-quantitative polymerase chain reaction (RT-qPCR) analysis. The isolation of total RNA and the production of cDNA was performed as previously described (21). qPCR was performed using a 7500 Real Time PCR System (Applied Biosystems; Thermo Fisher Scientific, Inc., Waltham, MA, USA) with a Two Step SYBR ${ }^{\circledR}$ PrimeScript $^{\text {TM }}$ RT-qPCR kit (Takara Bio, Inc., Otsu, Japan). The amplification was performed over 40 cycles with conditions of $95^{\circ} \mathrm{C}$ for $30 \mathrm{sec}$, $95^{\circ} \mathrm{C}$ for $5 \mathrm{sec}$ and $60^{\circ} \mathrm{C}$ for $34 \mathrm{sec}$. The relative quantitation of mRNA was analyzed using the $2^{-\Delta \Delta C q}$ method (22) and normalized by $\beta$-actin. The primers were as follows: $\beta$-actin sense, 5'-CATTGCTGACAGGATGCAGAAGG-3' and antisense, 5'-TGCTGGAAGGTGGACAGTGAGG-3'; p62 sense, 5'-GCTCTTCGGAAGTCAGCAAACC-3' and antisense, 5'-GCAGTTTCCCGACTCCATCTGT-3'.

Western blotting. Total protein from renal tissues was extracted using radio immunoprecipitation assay (RIPA) lysis buffer (1\% Triton X-100, $150 \mathrm{mM} \mathrm{NaCl}, 5 \mathrm{mM}$ EDTA, and $10 \mathrm{mM}$ Tris- $\mathrm{HCl}, \mathrm{pH}$ 7.0) containing a protease inhibitor cocktail (cat. no. WB-0071, Beijing Dingguo Changsheng Biotechnology Co. Ltd, Beijing, China). Proteins were quantified using a bicinchoninic acid kit. Equal amounts of protein from each sample (20-80 $\mu \mathrm{g}$ per lane) were mixed with SDS sample buffer, heated to $100^{\circ} \mathrm{C}$ for $10 \mathrm{~min}$ to expose epitopes and subjected to electrophoresis on a $10 \%$ SDS-PAGE gel. Proteins were transferred to a polyvinylidene fluoride membrane (EMD Millipore, Billerica, MA, USA). Following blocking in $2 \%$ bovine serum albumin (Gen-view Scientific Inc., USA) in TBS with Tween 20 for $1 \mathrm{~h}$ at room temperature, membranes were incubated with primary antibodies against p62 (cat. no. 5114, 1:1,000 dilution, Cell Signaling Technology, Inc., Danvers, MA, USA), LC-3B (cat. no. 3868, 1:1,000 dilution; Cell Signaling Technology, Inc.) and $\beta$-actin (cat. no. A1978, 1:2,000 dilution, Sigma-Aldrich; Merck KGaA, Darmstadt, Germany) at $4^{\circ} \mathrm{C}$ overnight. Following washing three times, the membranes were subsequently incubated with the HRP-conjugated goat anti-rabbit IgG (cat. no. BA1054, 1:3,000, Boster Biological Technology, Pleasanton, CA, USA) or goat anti-mouse IgG antibody (cat. no. BA1050, 1:3,000, Boster Biological Technology) for $1 \mathrm{~h}$ at room temperature. Bands were determined using the enhanced chemiluminescence method (cat. no. 170-5061, Bio-Rad Laboratories, Inc., Hercules, CA, USA). The levels of proteins were quantitatively analyzed using ImageJ software, version 1.48 (National Institutes of Health, Bethesda, MD, USA) and normalized to the $\beta$-actin band density.

Immunohistochemistry. Immunohistochemistry was performed using EnVision (TM) FLEX Mini kit, according to the manufacturer's protocol (cat. no. K8024, Dako; Agilent Technologies, Inc., Santa Clara, CA, USA). Briefly, slides were deparaffinized and hydrated in xylene and a graded series of alcohol. Heat-induced antigen retrieval was performed and endogenous peroxidase activity was blocked using a peroxidase-blocking reagent (as part of the EnVision FLEX Mini kit; cat. no. K8024-DM821, Dako; Agilent Technologies, Inc., Santa Clara, CA, USA). Sequentially, the slides were incubated with primary antibodies, HRP-conjugated secondary antibody (as part of the EnVision FLEX Mini kit; cat. no. K8024-DM822, Dako; Agilent Technologies, Inc.), diaminobenzidine and hematoxylin for $30 \mathrm{sec}$, at room temperature. Primary antibodies included p62 (cat. no. 5114, 1:50 dilution, Cell Signaling Technology, Inc.), Na-K-2Cl cotransporter (NKCC-2; cat. no. AB3562P, 1:200 dilution; EMD Millipore), aquaporin-2 (AQP2, cat. no. 3487S, 1:200 dilution, Cell Signaling Technology, Inc.), and aquaporin-1 (AQP1, cat. no. M00865, 1:50 dilution; Boster Biological Technology). Finally, the slides were analyzed using an optical microscope (Olympus Corporation, Tokyo, Japan).

Cell culture. The HK-2 cell line from the American Type Culture Collection (Manassas, VA, USA) was maintained in 
Dulbecco's modified Eagle's medium/F12 (Gibco; Thermo Fisher Scientific, Inc.) supplemented with $10 \%$ fetal bovine serum (Gibco; Thermo Fisher Scientific, Inc.) at $37^{\circ} \mathrm{C}$ in an incubator supplemented with $5 \% \mathrm{CO}_{2}$. Medium was renewed every 2 days.

Transfection of plasmids and siRNA into HK-2 cells. pENTER eukaryotic plasmids expressing p62 and small interfering RNA (siRNA) of p62 were purchased from Vigene Biosciences, Inc. (Rockville, MD, USA) and Shanghai GenePharma Co., Ltd. (Shanghai, China), respectively. The plasmid was extracted using Plasmid Maxprep kit (Vigorous Biotechnology, Beijing, China; www.vigorousbiol.com/), according to the manufacturer's protocol. Plasmid $(2 \mu \mathrm{g} / \mu \mathrm{l})$ and siRNA $(5 \mu \mathrm{l})$ transfections into HK-2 cells $\left(50,000\right.$ cells $\left./ \mathrm{cm}^{2}\right)$ were performed using Lipofectamine $3000^{\mathrm{TM}}$ (Invitrogen; Thermo Fisher Scientific, Inc.), according to the manufacturer's protocol. After $48 \mathrm{~h}$, protein was extracted for western blot analysis. The siRNA sequences were as follows: p62-siRNA sense, 5'-GAUCUG CGAUGGCUGCAAUTT-3' and antisence, 5'-AUUGCAGCC AUCGCAGAUCTT-3'; control-siRNA sense, 5'-UUCUCC GAACGUGUCACGUTT-3' and antisence, 5'-ACGUGACAC GUUCGGAGAATT-3'.

Cell grouping. Cells transfected with p62 overexpression plasmids/siRNA were divided into four groups: i) PBS+pENTER/negative;ii) PBS+p62-pENTER/p62-siRNA; iii)LPS+pENTER/negative;andiv)LPS+p62-pENTER/p62-siRNA groups. According to the preliminary results (23), cells $\left(50,000\right.$ cells $\left./ \mathrm{cm}^{2}\right)$ were treated with $1,000 \mathrm{ng} / \mathrm{ml}$ LPS for $12 \mathrm{~h}$. The cells and supernatants were collected for subsequent experiments.

Analysis of cell proliferation and lactate dehydrogenase $(L D H)$ level. The viability of cells cultured in 96-well plates $\left(4,000\right.$ cells $\left./ \mathrm{cm}^{2}\right)$ in each group was measured using a Cell Counting Kit-8 kit (Bimake, Shanghai, China; www. bimake.com/search?q=CCK-8), according to the manufacturer's protocol. The culture supernatants were used to measure the LDH level using an LDH cytotoxicity test kit (Nanjing Jiancheng Bioengineering Institute, Nanjing, China), according to the manufacturer's protocol.

Apoptosis detection using flow cytometry. The cells in each group were washed, digested and subjected to apoptosis analysis using an Annexin V-Fluorescein Isothiocyanate/Propidium Iodide Apoptosis Detection kit (BD Pharmingen; BD Biosciences, Franklin Lakes, NJ, USA), according to the manufacturer's protocol. Subsequently, flow cytometric analysis was performed using a flow cytometer (BD Biosciences). Data were analyzed using FlowJo software (version 7.6.5; FlowJo, LLC, Ashland, OR, USA).

Statistical analysis. All experiments were repeated at least three times. The quantitative data are presented as the mean \pm standard deviation. Comparisons among groups were analyzed using one-way analysis of variance with Tukey's post hoc test. Survival analysis was performed using the Kaplan-Meier method and the log-rank test. Statistical analysis was performed using GraphPad Prism 5 software (GraphPad
Software, Inc., La Jolla, CA, USA). P $<0.05$ was considered to indicate a statistically significant difference.

\section{Results}

Effects of LPS on the survival rate and renal function of mice. As presented in Fig. 1A, the survival rate of the LPS group was significantly decreased compared with the control group $(\mathrm{P}<0.05)$. LPS administration additionally decreased renal function at 8-24 h, as demonstrated by a significant increase in BUN and creatinine levels compared with the control group (Fig. 1B and C).

p62 and LC-3B expression in the kidneys of mice during endotoxemia. As indicated in Fig. 2A, renal p62 mRNA expression was apparent in the control group $(0 \mathrm{~h})$ compared with $\beta$-actin mRNA. The p62 signal was gradually decreased at $4 \mathrm{~h}$ subsequent to LPS injection, reaching the lowest level at $8 \mathrm{~h}$ and thereafter increasing at $12-24 \mathrm{~h}$. The protein expression pattern of p62 was similar to that of p62 mRNA (Fig. 2B and C). p62 protein was expressed at a relatively high level in normal renal tissues, gradually decreasing to the lowest level at $8 \mathrm{~h}$ following treatment with LPS, and subsequently increased over time. p62 protein expression was increased compared with the control group at $24 \mathrm{~h}$.

In order to clarify the effect of LPS on renal autophagy, the expression of the autophagy-associated protein LC3B was additionally examined. The ratio of LC3-II/LC3-I has been previously used to represent the level of autophagy (24). It was identified that the ratio of LC3B-II/LC3B-I was significantly increased at $4 \mathrm{~h}$ following treatment with LPS, reaching a peak at $8 \mathrm{~h}$, and was slightly decreased at $12 \mathrm{~h}$, although significantly higher than the control group. At $24 \mathrm{~h}$, the ratio was significantly reduced below the normal level (Fig. 2B and D).

Cellular localization of p62 protein in the renal cortex of mice during endotoxemia. The renal cellular localization of p62 in each group was determined by immunohistochemical staining at various time points following LPS injection. The results demonstrated that the expression pattern of p62 in mouse kidneys during endotoxemia was dynamic. In the control group $(0 \mathrm{~h})$, p62 protein was predominantly localized in the renal cortex (Fig. 3A). In addition, p62 was expressed primarily in renal cortical tubules with a small amount in the glomeruli (Fig. 3A).

In mice treated with LPS, the pattern of $\mathrm{p} 62$ protein expression in the renal cortex was consistent with findings from RT-qPCR analysis and western blotting, demonstrating minimal expression in mice treated with LPS for $8 \mathrm{~h}$ and increasing in mice treated with LPS for $24 \mathrm{~h}$ (Fig. 3B). Fig. 3C illustrates that AQP-1 was expressed primarily in proximal tubular segments in the renal cortex, and it was used as a marker of proximal tubules. Co-staining of kidney sections for p62 and AQP-1 confirmed the localization of p62 in the proximal tubules of the renal cortex. In addition, p62 protein was primarily detected in the cytoplasm of the cortical tubules, and little was detected in the nucleus.

Expression and cellular localization of p62 protein in the renal medullas of mice during endotoxemia. The expression and cellular localization of p62 protein in renal medullas 

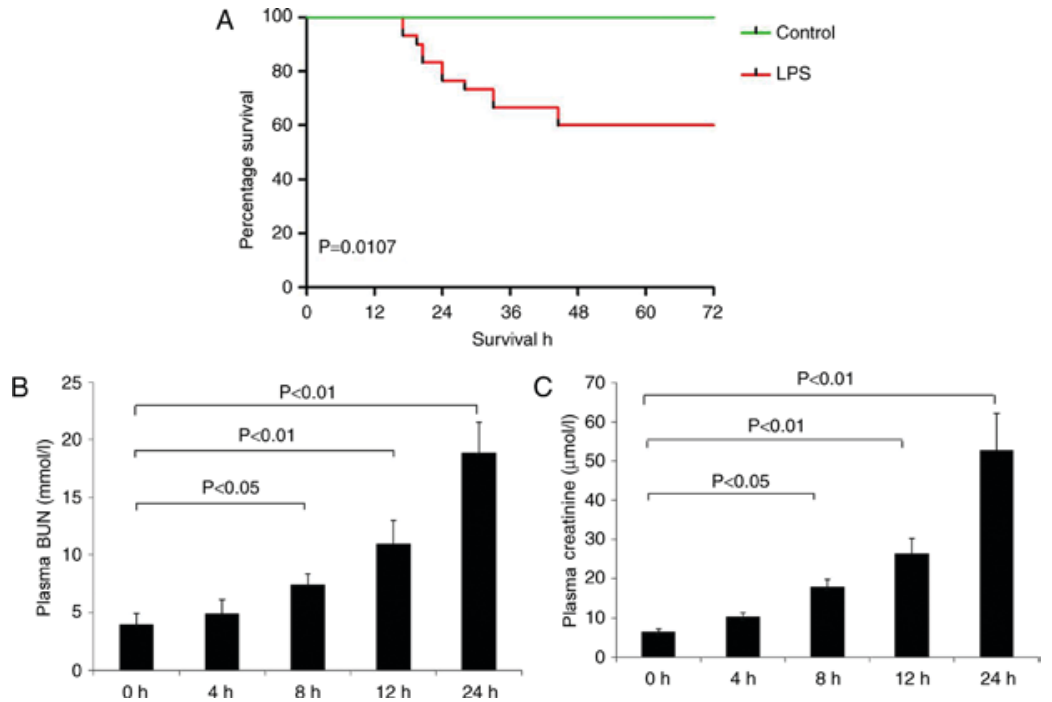

Figure 1. Effects of LPS on the survival rate and renal function of mice. (A) The survival curves were plotted using GraphPad Prism 5 software. The survival rate of the LPS group was $60 \%(n=20)$, significantly decreased compared with the saline group $(n=10 ; P<0.05)$. (B) BUN and $(C)$ creatinine measurements in serum were performed at 0-24 $\mathrm{h}$ following treatment with LPS. The LPS groups (8-24 h) exhibited a significant increase in BUN and creatinine levels compared with the control group. $n \geq 6$. Data are presented as the mean \pm standard deviation. LPS, lipopolysaccharide; BUN, blood urea nitrogen.
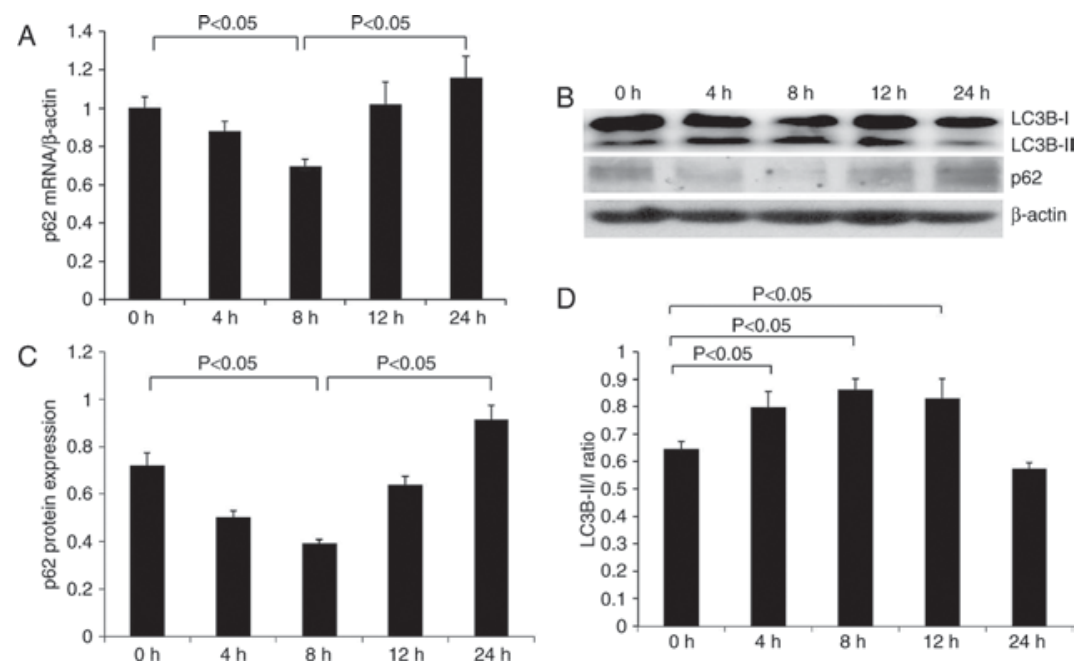

Figure 2. Expression of p62 and LC-3B in the whole kidneys of endotoxemic mice. (A) Renal p62 mRNA expression was detected in the whole kidneys of mice at various time points by reverse transcription-quantitative polymerase chain reaction analysis. (B) p62 and LC-3B protein expression was detected in the whole kidneys of mice at various time points by western blotting. (C) Densitometric analysis of p62 expression. (D) Densitometric analysis of LC-3B protein. $\mathrm{n} \geq 6$. Data are presented as the mean \pm standard deviation. $\mathrm{p} 62$, sequestome-1; LC-3B, light chain-3B.

was LPS-dependent. From $8 \mathrm{~h}$ following treatment with LPS, it was observed that p62 was redistributed from the cortex to the outer medullas (Fig. 4A). In order to determine the exact location of $\mathrm{p} 62$, the present study detected the expression of p62 and NKCC-2 (a marker of the thick ascending limb) using consecutive kidney sections at $8 \mathrm{~h}$ following LPS administration. The results demonstrated that p62 and NKCC-2 were co-localized, confirming that p62 was located in the thick ascending limb (Fig. 4B). In addition, as a marker of collecting ducts, AQP2 was additionally detected using consecutive sections. By contrast, the AQP2-positive regions exhibited a weak p62 signal (Fig. 4C), indicating substantially decreased p62 expression in the collecting ducts in the outer medullas. It was unexpected that $\mathrm{p} 62$ protein was primarily detected in the nuclei of tubular cells in outer medulla.
The redistribution of $\mathrm{p} 62$ in the inner medulla (Fig. 5A) was different compared with that in the outer medulla. In control mice, the expression of p62 was low in the inner medullas. However, in mice treated with LPS, high levels of p62 were detected in the nuclei of tubular cells in the inner medulla (Fig. 5A) from $12 \mathrm{~h}$. Co-staining of kidney sections for $\mathrm{p} 62$ and AQP-2 confirmed the accumulation of p62 in collecting ducts (Fig. 5B). The low level of p62 in AQP-1 (additionally a marker of the thin limb of the loops of Henle)-positive cells (Fig. 5C) indicated that little p62 protein was accumulated in the thin limb of the loops of Henle.

In addition, the protein level of p62 in medullary tissue was examined using western blotting. Fig. 6 illustrates the increased level of p62 in LPS-treated mice compared with control mice. The level of $\mathrm{p} 62$ reached a maximum level at $12 \mathrm{~h}$, which was maintained until $24 \mathrm{~h}$. 
A

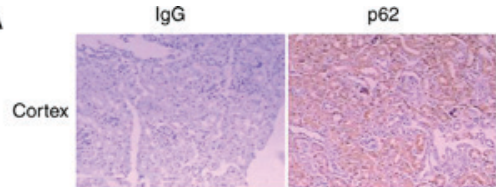

B

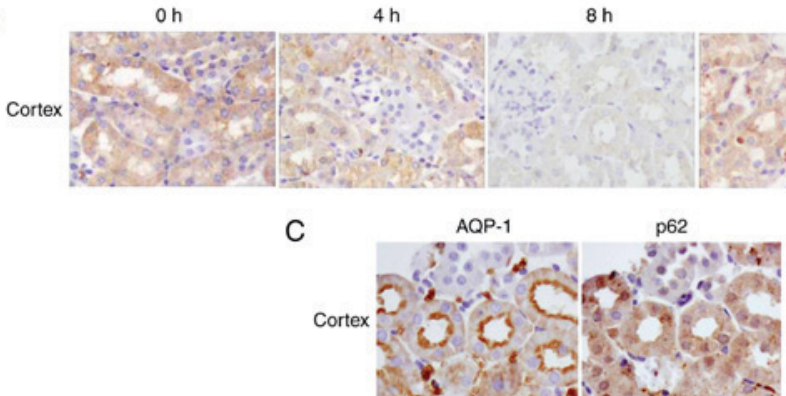

Figure 3. Localization of p62 protein in the renal cortex of mice during endotoxemia. (A) Immunostaining of kidney slides with anti-p62 and anti-IgG antibody in the renal cortex of normal controls (magnification, $\mathrm{x} 40$ ). (B) The localization of p62 protein in the renal cortex of mice was measured by immunostaining at various time points (magnification, x200). (C) Double-immunohistochemical staining of p62 and AQP-1 in consecutive kidney slides in normal mice (magnification, $\mathrm{x} 400$ ). $\mathrm{n} \geq 6$. IgG, immunoglobulin G; p62, sequestome-1; AQP-1, aquaporin-1.

A

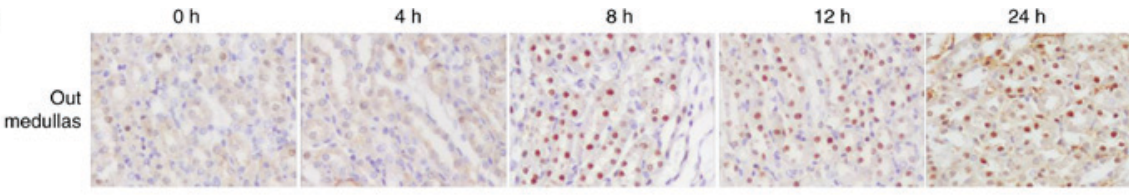

B

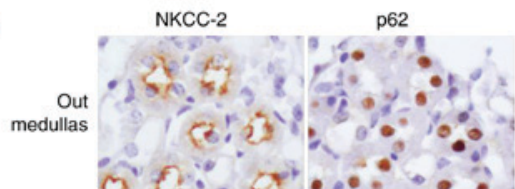

C

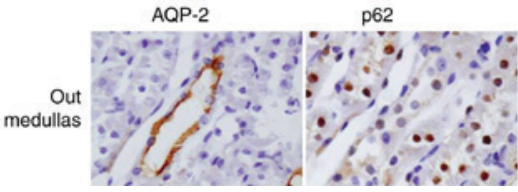

Figure 4. Localization of p62 protein in the outer medullas of mice during endotoxemia. (A) The localization of p62 protein in the outer medullas of mice was measured by immunostaining at various time points (magnification, x200). (B) Double-immunohistochemical staining of NKCC-2 and p62 in consecutive kidney slides at $8 \mathrm{~h}$ following treatment with LPS (magnification, x400). (C) Double-immunohistochemical staining of AQP-2 and p62 in consecutive kidney slides at $8 \mathrm{~h}$ following treatment with LPS (magnification, $\mathrm{x} 400$ ). $\mathrm{n} \geq 6$. Out, outer; $\mathrm{p} 62$, sequestome-1; NKCC-2, Na-K-2Cl cotransporter; AQP2, aquaporin-2; LPS, lipopolysaccharide.

A $\mathrm{Oh}$ $4 \mathrm{~h}$ $8 \mathrm{~h}$ $12 \mathrm{~h}$ $24 \mathrm{~h}$

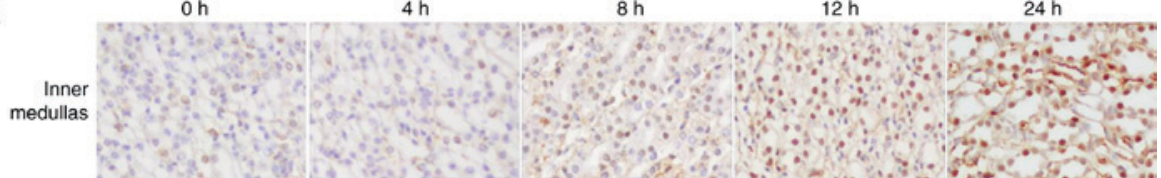

B

AQP-2

p62

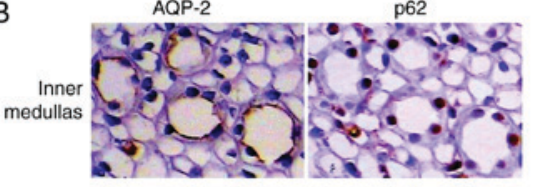

C

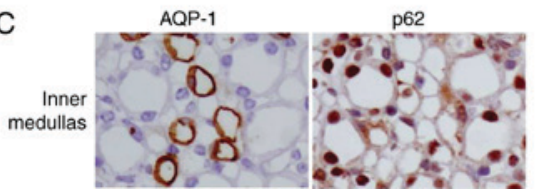

Figure 5. Localization of p62 protein in the inner medullas of mice during endotoxemia. (A) Localization of p62 protein in the inner medullas of mice was detected by immunostaining at various time points (magnification, x200). (B) Double-immunohistochemical staining of p62 and AQP-2 in consecutive kidney slides at $12 \mathrm{~h}$ following treatment with LPS (magnification, $\mathrm{x} 400$ ). (C) Double-immunohistochemical staining of $\mathrm{p} 62$ and AQP-1 on consecutive kidney slides at $12 \mathrm{~h}$ subsequent to treatment with LPS (magnification, $\mathrm{x} 400$ ). $\mathrm{n} \geq 6$. p62, sequestome-1; AQP, aquaporin; LPS, lipopolysaccharide. 

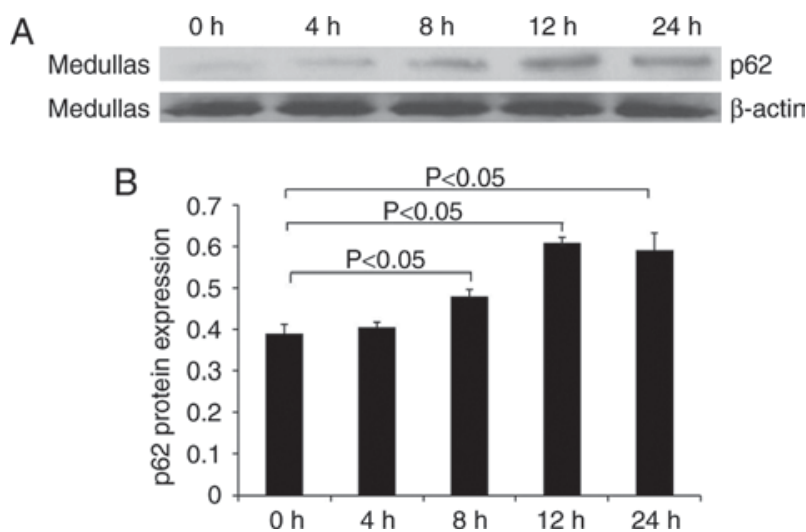

Figure 6. p62 protein expression in renal medullas of endotoxemic mice. p62 protein expression in renal medullas was detected in mice at various time points using (A) western blotting and (B) densitometric analysis. $n \geq 6$. Data are presented as the mean \pm standard deviation. p62, sequestome- 1 .

Effects of p62 overexpression on LPS-induced injury in the renal tubular epithelial cell line $H K-2$. Western blotting results demonstrated that the levels of p62 protein were significantly increased in HK-2 cells transfected with p62 plasmid (Fig. 7A). In addition, these cells were treated with LPS to explore the effects of p62 on LPS-induced viability, LDH level and apoptosis. The results demonstrated that p62 overexpression significantly decreased cell viability and increased LDH release (Fig. 7B and C). Flow cytometry analysis demonstrated that p62 overexpression significantly increased the apoptosis of HK-2 cells (Fig. 7D).

Effects of interference with p62 expression on LPS-induced injury in the renal tubular epithelial cell line $H K-2$. The expression of p62 was significantly inhibited in HK-2 cells transfected with p62 siRNA at the protein level (Fig. 8A). In contrast to the overexpression of p62 in HK-2 cells, p62 downregulation significantly increased cell viability, and decreased LDH release (Fig. 8B and C). Flow cytometry demonstrated that p62 downregulation significantly decreased the apoptosis of HK-2 cells induced by LPS (Fig. 8D). These results demonstrated that p62 is able to promote apoptosis and the release of the toxic substance LDH, and to inhibit the proliferation of renal tubular epithelial cells.

\section{Discussion}

The overall 72-h survival rate and the renal functional parameters indicated that the AKI model was successfully established. In the present study, the renal expression and location of p62 was examined in a mouse model of endotoxemia induced via injection of LPS. In mice treated with LPS, the protein levels of p62 significantly decreased and subsequently increased. Previous studies have demonstrated that p62 is one of the selective mammalian autophagy cargo receptors (13). Under normal conditions, p62 is incorporated into the autophagosome and degraded by autophagy (25). Certain stimuli, including hunger and hypoxia, may increase autophagy, which may induce p62 degradation, resulting in decreased intracellular levels of p62 (26). Therefore, the expression of p62 may indirectly represent the level of autophagy. The decreased expression of p62 protein and the increased expression of LC3-II protein in the results of the present study indicated that autophagy was induced in the kidneys of LPS-treated mice, suggesting that autophagy may serve a role in the mechanism of LPS-induced AKI. A significant decrease in the mRNA levels of p62 was additionally observed in the kidneys of mice treated with LPS. The results of the present study demonstrated the inhibited transcription and production of p62 in the kidney at the early stages of endotoxemia, in addition to the degradation of p62, which contributed to the reduction of p62 in LPS-induced AKI. In addition, the expression level of p62, whether mRNA or protein, was demonstrated to increase gradually and was increased compared with the normal level at $24 \mathrm{~h}$. It was suggested that p62 may serve as an autophagy substrate and may serve a role in septic AKI. It was reported that the accumulation of p62 may additionally activate nuclear factor- $\kappa \mathrm{B}$, increase interleukin- $1 \beta$ production, and promote the activation of caspase- 1 and caspase- 8 , which aggravate inflammation and apoptosis $(27,28)$. However, whether p62 may serve a role by regulating signaling pathways in LPS-induced AKI remains unknown.

Immunohistochemistry indicated that p62 protein was predominantly located in the cytoplasm of proximal tubules in the renal cortex under normal conditions. Consistent with the expression of p62 in the whole kidney demonstrated by RT-qPCR analysis and western blotting, p62 was observed to be decreased in the cytoplasm of proximal tubules, followed by an increase with a longer treatment with LPS. A previous study demonstrated that the LIR domain enables p62 to combine with LC3-II, and the UBA domain enables p62 to bind to ubiquitin; consequently, ubiquitinated proteins may be degraded by autophagy $(8,29)$. Therefore, the intracellular level of p62 is regulated by autophagy through the interaction of LC3-II with p62. Previously, the LPS-induced expression of LC3-II was detected in mouse kidneys $(30,31)$. Consistently, the results of the present study demonstrated that LC3-II in the kidneys increased during treatment with LPS, reaching a peak at 8-12 h; thereafter, it decreased at $24 \mathrm{~h}$. Despite the distinct time frame of the expression of renal p62 and LC3-II, they exhibit the same cellular localization (32). Following treatment with LPS, the expression of LC3-II in the proximal tubules of the renal cortex was significantly increased compared with normal mice, indicating the formation of autophagosomes (30). However, the expression of LC3-II in the renal medulla was not notably increased (30). The results of the present study demonstrated that autophagy was predominantly induced in the proximal tubules of the renal cortex during LPS-induced AKI.

AQP-1 is a marker of proximal tubules. Co-localization analysis of p62 and AQP-1 at proximal tubules using immunohistochemistry demonstrated that p62 was localized in the cytoplasm of renal tubular epithelial cells. Therefore, the roles of p62 were examine using the renal tubular epithelial cell line HK-2. The results of the present study demonstrated that the overexpression of p62 aggravated LPS-induced injury to renal tubular epithelial cells, however, interference with p62 expression decreased the damage to HK-2 cells. As a multifunctional protein, p62 may be upregulated via autophagy deficiency or inhibition (10). The inhibition of autophagy may upregulate the accumulation of p62 and enhance caspase-8-dependent apoptotic pathways (33-35). However, the role of p62 in 
A
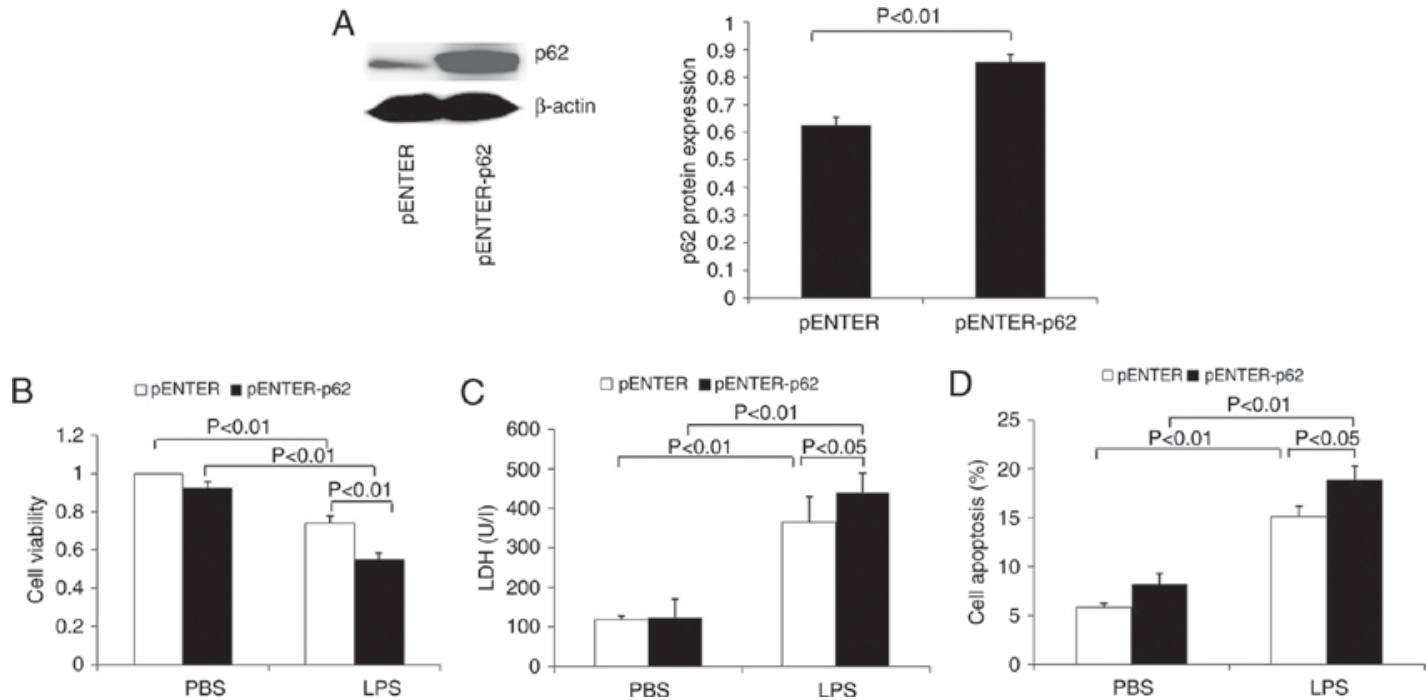

Figure 7. Effects of p62 overexpression on injury in renal tubular epithelial cells treated with LPS. (A) The expression of p62 protein in HK-2 cells transfected with control plasmid (pENTER) and p62 plasmid (pENTER-p62) following treatment with 1,000 $\mathrm{ng} / \mathrm{ml} \mathrm{LPS} \mathrm{for} 12 \mathrm{~h}$ measured by western blotting (left panel). The right panel exhibits the ratios of p62/ $\beta$-actin. (B) Cell viability of different groups measured using a Cell Counting Kit- 8 kit ( $n=3$ ). (C) LDH cytotoxicity in the different groups $(n=3)$. (D) Ratios of cellular apoptosis $(n=3)$. Data are presented as the mean \pm standard deviation. $p 62$, sequestome-1; LDH, lactate dehydrogenase; LPS, lipopolysaccharide.

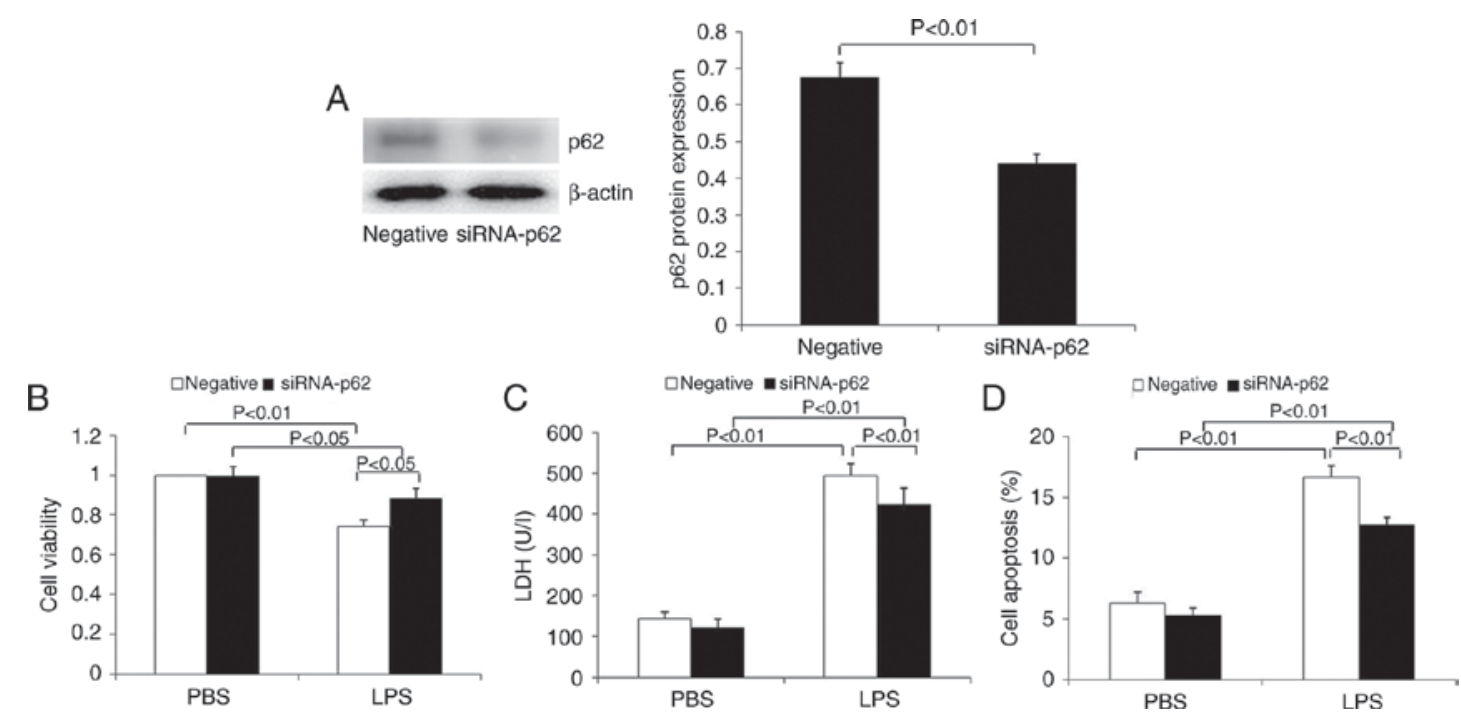

Figure 8. Effects of p62 interference on injury in renal tubular epithelial cells treated with LPS. (A) The levels of p62 expression in renal tubular epithelial cells transfected with control siRNA and siRNA-p62 following treatment with 1,000 ng/ml LPS for $12 \mathrm{~h}$ measured by western blotting (left panel). The right panel exhibits the ratios of p62/ $\beta$-actin. (B) The viabilities of HK-2 cells in the different groups (n=3) measured using a Cell Counting Kit- 8 kit. (C) LDH cytotoxicity detection in the different groups $(n=3)$. (D) The ratios of apoptotic cells in the different groups $(n=3)$. Data are presented as the mean \pm standard deviation. p62, sequestome-1; LDH, lactate dehydrogenase; LPS, lipopolysaccharide; siRNA, small interfering RNA.

LPS-induced AKI remains largely unknown. The results of the present study demonstrated that the overexpression of p62 promoted the apoptosis of renal tubular epithelial cells. In combination with the results of the animal experiments, it was indicated that the degradation of p62 by activated autophagy at the early stages of endotoxemia contributed to the inhibition of apoptosis; however, at the late stages of endotoxemia, inhibition or dysfunction of autophagy resulted in p62 accumulation, which increased the apoptosis of renal tubular epithelial cells and accelerated renal injury.

In the present study, it was unexpected that $\mathrm{p} 62$ was observed to redistribute to the nucleus of the tubule cells in the medulla from $8 \mathrm{~h}$ subsequent to treatment with LPS. p62 has generally been considered to be a cytosolic protein, and little attention has been paid to the possible nuclear roles of this protein. In eukaryotic cells, molecular trafficking between the nucleus and the cytoplasm is highly regulated via cellular homeostasis and signaling (36). However, such regulation may be disturbed under stress through the disruption of nucleocytoplasmic transport pathways, resulting in the nucleocytoplasmic redistribution of a number of functional proteins (36). Consistent with the results of the present study, Pankiv et al (37) demonstrated that p62 may be shuttled between the cytoplasm and nucleus. p62 contains two important domains, the nuclear localization signal and 
nuclear export signal domains, which may facilitate the shuttling of p62 between the cytoplasm and nucleus. Additionally, Pankiv et al (37) additionally discovered that p62 may recruit nuclear polyubiquitinated proteins or protein aggregates to promyelocytic leukemia bodies and may aid in their proteasomal degradation in the nucleus, indicating the potential nuclear roles of this protein. However, the function of p62 transported into the nucleus during endotoxemia is unknown.

At present, the majority of studies have focused on the association between cytoplasmic proteins and AKI. Nuclear autophagy has rarely been reported. Whether the increased expression of p62 in the nucleus of renal medullar cells during LPS-induced AKI is associated with nuclear autophagy remains unknown. In addition to mediating selective autophagy as an autophagy adaptor protein, p62 may act as an important signaling hub to control cell survival and apoptosis $(38,39)$. For example, p62 may promote apoptosis by mediating the aggregation of caspase-8 (39). Whether the enhanced p62 expression observed in the present study may promote the apoptosis of medullar cells by activating an apoptotic pathway remains unknown. Therefore, further studies are required to better understand the role of p62 in LPS-induced AKI.

In conclusion, the mRNA and protein levels of p62 decreased in the kidney during early inflammation and subsequently increased at $24 \mathrm{~h}$ following treatment with LPS. Immunohistochemistry indicated that p62 protein was predominantly expressed in the cytoplasm of renal tubules in control mice, although it was redistributed to the medullas following LPS injection. In vitro experiments demonstrated that p62 overexpression was able to aggravate LPS-induced injury in renal tubular epithelial cells. The results of the present study supported a potential role of p62 in the kidney during LPS-stimulated endotoxemia via alterations in the expression level and location of p62. Further studies are required to elucidate the function of p62 in the cytoplasm and nucleus of renal tubular epithelial cells during endotoxemia.

\section{Acknowledgements}

Not applicable.

\section{Funding}

The present study was supported by the National Natural Science Foundation of China (grant nos. 81571880, 81373147, 81671895, 30901555 and 30972870), the Natural Science Foundation of Hunan Province (grant no. 2016JJ2157) and the Changzhi Medical College Innovation Team Project (grant no. CX201501).

\section{Availability of data and materials}

The analyzed data sets generated during the study are available from the corresponding author on reasonable request.

\section{Authors' contributions}

TL, JZ, YL and XX conceived and designed the experiments. TL, JZ, SM and YX executed the experiments and analyzed the data. TL wrote manuscript. All authors interpreted the data, critically revised the manuscript for important intellectual contents and approved the final version.

\section{Ethics approval and consent to participate}

The present study was approved by the Ethical Committee of the Animal Experimental Institute of Central South University, Changsha, China, and carried out in accordance with the approved protocol.

\section{Consent for publication}

Not applicable.

\section{Competing interests}

The authors declare that they have no competing interests.

\section{References}

1. Mehta RL, Bouchard J, Soroko SB, Ikizler TA, Paganini EP, Chertow GM and Himmelfarb J; Program to Improve Care in Acute Renal Disease (PICARD) Study Group: Sepsis as a cause and consequence of acute kidney injury: Program to improve care in acute renal disease. Intensive Care Med 37: 241-248, 2011.

2. Lopes JA, Jorge S, Resina C, Santos C, Pereira A, Neves J, Antunes F and Prata MM: Acute kidney injury in patients with sepsis: A contemporary analysis. Int J Infect Dis 13: 176-181, 2009.

3. Cruz MG, Dantas JG, Levi TM, Rocha Mde S, de Souza SP, Boa-Sorte N, de Moura CG and Cruz CM: Septic versus non-septic acute kidney injury in critically ill patients: Characteristics and clinical outcomes. Rev Bras Ter Intensiva 26: 384-391, 2014.

4. Choi AM, Ryter SW and Levine B: Autophagy in human health and disease. N Engl J Med 368: 651-662, 2013.

5. Jiang M, Liu K, Luo J and Dong Z: Autophagy is a renoprotective mechanism during in vitro hypoxia and in vivo ischemia-reperfusion injury. Am J Pathol 176: 1181-1192, 2010.

6. Kimura T, Takabatake Y, Takahashi A, Kaimori JY, Matsui I, Namba T, Kitamura H, Niimura F, Matsusaka T, Soga T, et al: Autophagy protects the proximal tubule from degeneration and acute ischemic injury. J Am Soc Nephrol 22: 902-913, 2011.

7. Leventhal JS, Ni J, Osmond M, Lee K, Gusella GL, Salem F and Ross MJ: Autophagy limits endotoxemic acute kidney injury and alters renal tubular epithelial cell cytokine expression. PLoS One 11: e0150001, 2016.

8. Komatsu M and Ichimura Y: Physiological significance of selective degradation of P62 by autophagy. FEBS Lett 584: 1374-1378, 2010.

9. Shaid S, Brandts $\mathrm{CH}$, Serve $\mathrm{H}$ and Dikic I: Ubiquitination and selective autophagy. Cell Death Differ 20: 21-30, 2013.

10. Moscat J and Diaz-Meco MT: P62 at the crossroads of autophagy, apoptosis, and cancer. Cell 137: 1001-1004, 2009.

11. Lippai M and Low P: The role of the selective adaptor P62 and ubiquitin-like proteins in autophagy. Biomed Res Int 2014: $832704,2014$.

12. DeVorkin L and Gorski SM: Monitoring autophagic flux using Ref(2)P, the Drosophila P62 ortholog. Cold Spring Harb Protoc 2014: 959-966, 2014.

13. Lin X, Li S, Zhao Y, Ma X, Zhang K, He X and Wang Z: Interaction domains of P62: A bridge between P62 and selective autophagy. DNA Cell Biol 32: 220-227, 2013.

14. Pankiv S, Clausen TH, Lamark T, Brech A, Bruun JA, Outzen H, Øvervatn A, Bjørkøy G and Johansen T: P62/SQSTM1 binds directly to Atg8/LC3 to facilitate degradation of ubiquitinated protein aggregates by autophagy. J Biol Chem 282: 24131-24145, 2007.

15. Rogov V, Dötsch V, Johansen T and Kirkin V: Interactions between autophagy receptors and ubiquitin-like proteins form the molecular basis for selective autophagy. Mol Cell 53: 167-178, 2014.

16. Manley S, Williams JA and Ding WX: Role of p62/SQSTM1 in liver physiology and pathogenesis. Exp Biol Med (Maywood) 238: $525-538,2013$. 
17. Luo RZ, Yuan ZY, Li M, Xi SY, Fu J and He J: Accumulation of $\mathrm{P} 62$ is associated with poor prognosis in patients with triple-negative breast cancer. Onco Targets Ther 6: 883-888, 2013.

18. Laurin N, Brown JP, Morissette J and Raymond V: Recurrent mutation of the gene encoding sequestosome 1 (SQSTM1/p62) in paget disease of bone. Am J Hum Genet 70: 1582-1588, 2002.

19. Yang L, Li P, Fu S, Calay ES and Hotamisligil GS: Defective hepatic autophagy in obesity promotes ER stress and causes insulin resistance. Cell Metab 11: 467-478, 2010

20. Codogno P and Meijer AJ: Autophagy: A potential link between obesity and insulin resistance. Cell Metab 11: 449-451, 2010

21. Liu Y, Jia Z, Sun Y, Zhou L, Downton M, Chen R, Zhang A and Yang T: Postnatal regulation of 15-hydroxyprostaglandin dehydrogenase in the rat kidney. Am J Physiol Renal Physiol 307: F388-F395, 2014.

22. Livak KJ and Schmittgen TD: Analysis of relative gene expression data using real-time quantitative PCR and the 2(-Delta Delta C(T)) method. Methods 25: 402-408, 2001.

23. Li T, Liu Y, Zhao J, Miao S, Xu Y, Liu K, Liu M, Wang G and Xiao X: Aggravation of acute kidney injury by mPGES-2 down regulation is associated with autophagy inhibition and enhanced apoptosis. Sci Rep 7: 10247, 2017.

24. Kadowaki M and Karim MR: Cytosolic LC3 ratio as a quantitative index of macroautophagy. Methods Enzymol 452: 199-213, 2009.

25. Jiang P and Mizushima N: LC3- and P62-based biochemica methods for the analysis of autophagy progression in mammalian cells. Methods 75: 13-18, 2015.

26. Klionsky DJ, Abdalla FC, Abeliovich H, Abraham RT, Acevedo-Arozena A, Adeli K, Agholme L, Agnello M Agostinis P, Aguirre-Ghiso JA, et al: Guidelines for the use and interpretation of assays for monitoring autophagy. Autophagy 8: 445-544, 2012

27. Choe JY, Jung HY, Park KY and Kim SK: Enhanced P62 expression through impaired proteasomal degradation is involved in caspase-1 activation in monosodium urate crystal-induced interleukin-1b expression. Rheumatology (Oxford) 53: 1043-1053, 2014.

28. Kovacs JR, Li C, Yang Q, Li G, Garcia IG, Ju S, Roodman DG, Windle JJ, Zhang $\mathrm{X}$ and Lu B: Autophagy promotes T-cell survival through degradation of proteins of the cell death machinery. Cell Death Differ 19: 144-152, 2012.

29. Komatsu M, Kageyama S and Ichimura Y: P62/SQSTM1/A170 Physiology and pathology. Pharmacol Res 66: 457-462, 2012.
30. Wu Y, Zhang Y, Wang L, Diao Z and Liu W: The role of autophagy in kidney inflammatory injury via the NF- $\kappa B$ route induced by LPS. Int J Med Sci 12: 655-667, 2015.

31. Mei S, Livingston M, Hao J, Li L, Mei C and Dong Z: Autophagy is activated to protect against endotoxic acute kidney injury. Sci Rep 6: 22171, 2016.

32. Sansanwal P and Sarwal MM: P62/SQSTM1 prominently accumulates in renal proximal tubules in nephropathic cystinosis. Pediatr Nephrol 27: 2137-2144, 2012.

33. Han J, Hou W, Goldstein LA, Lu C, Stolz DB, Yin XM and Rabinowich H: Involvement of protective autophagy in TRAIL resistance of apoptosis-defective tumor cells. J Biol Chem 283: 19665-19677, 2008.

34. Huang S and Sinicrope FA: Celecoxib-induced apoptosis is enhanced by ABT-737 and by inhibition of autophagy in human colorectal cancer cells. Autophagy 6: 256-269, 2010.

35. Huang S, Okamoto K, Yu C and Sinicrope FA: P62/sequestosome-1 up-regulation promotes ABT-263-induced caspase- 8 aggregation/activation on the autophagosome. J Biol Chem 288: 33654-33666, 2013.

36. Kose $\mathrm{S}$ and Imamoto $\mathrm{N}$ : Nucleocytoplasmic transport under stress conditions and its role in HSP70 chaperone systems. Biochim Biophys Acta 1840: 2953-2960, 2014.

37. Pankiv S, Lamark T, Bruun JA, Øvervatn A, Bjørkøy G and Johansen T: Nucleocytoplasmic shuttling of p62/SQSTM1 and its role in recruitment of nuclear polyubiquitinated proteins to promyelocytic leukemia bodies. J Biol Chem 285: 5941-5953, 2010.

38. Sanz L, Diaz-Meco MT, Nakano H and Moscat J: The atypical PKC-interacting protein P62 channels NF-kappaB activation by the IL-1-TRAF6 pathway. EMBO J 19: 1576-1586, 2000.

39. Jin Z, Li Y, Pitti R, Lawrence D, Pham VC, Lill JR and Ashkenazi A: Cullin3-based polyubiquitination and P62-dependent aggregation of caspase-8 mediate extrinsic apoptosis signaling. Cell 137: 721-735, 2009. International (CC BY-NC-ND 4.0) License. 